\title{
Importancia de la inteligencia emocional y su impacto en la vida de los estudiantes de la educación superior
}

\author{
Importance of emotional intelligence and its impact on the life of higher education students \\ Miriam Alegría-Alegría ${ }^{a}$,Dulce M. Sánchez-Zúñiga ${ }^{b}$
}

\begin{abstract}
:
The main purpose of this work focuses on the great importance that emotional intelligence has gained in recent years and the way, that Goleman (1996) indicates, the union of reason and emotion is essential to understand the development of human intelligence. Moreover, various studies show the importance of developing emotional competencies in the educational context, and more specifically in university students, in our work pretends to make clear in the first instance the relationship stress and emotions, later we present a summary from a physiological view of how the body responds to emotions. We will approach the elements that make up emotional intelligence, all this in order to be able to contribute to the importance and attention that is currently paid to emotional education in high institutions: its value for the life and training of students and for its success. personal and professional.
\end{abstract}

\section{Keywords:}

Emotional intelligence, emotions, university students, education, stress

\begin{abstract}
Resumen:
El propósito principal de este trabajo se centra en la gran importancia que ha cobrado la inteligencia emocional en los últimos años y como, según indica Goleman (1996), la unión de la razón y la emoción es fundamental para comprender el desarrollo de la inteligencia humana. Además, diversos estudios muestran la importancia de desarrollar competencias emocionales en el contexto educativo, y más concretamente en los estudiantes universitarios, en nuestro trabajo se pretende dejar claro en primera instancia la relación estrés y las emociones, posteriormente presentamos un resumen desde una vista fisiológica de cómo el organismo responde ante las emociones. Abordaremos los elementos que componen a la inteligencia emocional, todo esto con el fin de poder contribuir a la importancia y atención que se presta actualmente a la educación emocional en las instituciones superiores: su valor para la vida y formación de los alumnos y para su éxito personal y profesional.
\end{abstract}

\section{Palabras Clave:}

Inteligencia emocional, emociones, universitarios, educación, estrés

\section{Introducción}

"El control de la vida emocional y su subordinación a un objetivo resulta esencial para espolear y mantener la atención, la motivación y la creatividad". -Daniel Goleman.

Las actividades académicas universitarias, constituyen una gran fuente de estrés y ansiedad para los estudiantes, estos niveles altos de estrés provocan desequilibrios emocionales que a la vez pueden llegar a provocar afecciones a nivel psicológico y sin duda altas repercusiones en su salud mental, que a la larga afectará completamente la salud abarcando desde lo físico hasta lo mental y social. La mayoría de las personas sabemos que el estrés afecta de una manera directa nuestra salud, pero ¿qué hay acerca de las emociones? Escuchar sobre el estrés y su importante repercusión en la salud es común, pero ¿cómo hace el estrés para afectar?, el estrés es un estado en el que se busca estar activo, al estar más activados abrimos una puerta a las reacciones

\footnotetext{
Miriam Alegría Alegría, Universidad Autónoma del Estado de Hidalgo, https://orcid.org/0000-0001-8679-7070, Email: al419328@uaeh.edu.mx

b Dulce María Sánchez Zúñiga, Universidad Autónoma del Estado de Hidalgo, https://orcid.org/0000-0001-6002-6497, Email: sa343404@uaeh.edu.mx
} 
emocionales negativas, especialmente a la ansiedad y en menor medida a otras, como la ira. A largo plazo, todo esto se convierte en ansiedad y en tristeza o depresión. Las emociones además de alterar la forma en que se manejan las situaciones se ha demostrado que afecta el desempeño académico de los estudiantes y sus relaciones sociales. El estrés va afectar potencialmente nuestra salud emocional, partiendo de lo anterior es para nosotros de gran interés poder analizar y conocer, cómo es que las emociones tienen un papel tan importante en nuestra salud, por ello es que recurrimos al concepto inteligencia emocional, el cual nos ayuda a entender de qué manera podemos influir de un modo adaptativo e inteligente tanto sobre nuestras emociones como en nuestra interpretación de los estados emocionales de los demás, se ha pasado a considerarque las emociones son algo intrínseco a nuestro comportamiento gran parte de nuestras decisiones son influenciadas en mayor o menor grado por las emociones, por ejemplo podríamos plantearnos, ¿Elegí a mi pareja porque era objetivamente la mejor opción?. Siempre hemos oído decir que el Cociente intelectual es un buen indicador para saber si una persona será exitosa en la vida, esto no es en sí incorrecto, pero, de hecho, los investigadores y las corporaciones empezaron a detectar hace unas décadas que las capacidades y habilidades necesarias para tener éxito en la vida eran otras que iban más allá del uso de la lógica y la racionalidad, para esto es necesario tener en cuenta una concepción más amplia de lo que son las habilidades cognitivas básicas, aquello que entendemos que es la inteligencia, cabe resaltar que existen personas con un dominio emocional mucho más desarrollado que otras, aquí podríamos ejemplificar con un estereotipo de estudiante intelectual capaz de memorizar datos y llegar a las mejores soluciones lógicas, pero con una vida emocional y sentimental vacía. Por otro lado, podemos encontrar personas cuyas capacidades intelectuales son muy limitadas, pero en cambio consiguen tener una vida exitosa en lo que refiere al ámbito sentimental, e incluso en el profesional.

Este par de ejemplos llevados al extremo son poco habituales, pero sirven para percatarse de que es necesario prestar más atención a esta clase de habilidades emocionales, que pueden marcar nuestra vida y nuestra felicidad tanto o más que nuestra capacidad para puntuar alto en una prueba de inteligencia convencional.

\section{Desarrollo}

El siglo XX se caracteriza por el auge y expansión del término "Inteligencia Emocional". Se define la inteligencia emocional como "la capacidad de reconocer nuestros propios sentimientos y los de los demás, de motivarnos y de manejar adecuadamente las relaciones" Daniel Goleman (1995). Es una herramienta básica que utilizamos los seres humanos para llevar un control de las respuestas que ejerceremos ante las situaciones que nos enfrentamos día con día. Es el medio que se posee para la interacción con los otros y permitir el establecimiento de relaciones sin afectar a los otros a través de las palabras o acciones que realicemos en dicha actividad social. Este tipo de inteligencia, según Goleman (1996), se refleja en la forma de interacción de las personas con el mundo que les rodea y tiene dos componentes, muy relacionados con dos tipos de inteligencias según la Teoría de las Inteligencias Múltiples de Gardner (2005): el componente intrapersonal, relacionado con las capacidades para de identificación, percepción y dominio de las emociones en uno mismo, manifestándose a través de las autoconciencia y el autocontrol y el componente interpersonal, vinculado con las capacidades de identificación y percepción de emociones en otras personas (definido como "empatía") y la capacidad de relacionarnos de manera positiva de forma social (poseer "habilidades sociales"). Además, la inteligencia emocional tendría diversos objetivos, entre los que destacan: ser capaz de conocer las emociones del propio individuo en el momento en el que surgen; tener la capacidad de controlar las propias emociones y acomodarlas a la situación en la que se producen; reconocer, no sólo las propias emociones, sino también las emociones de las personas que nos rodean, favoreciendo la empatía: mantener un control emocional, teniendo la capacidad de desarrollar la motivación; controlar, favorecery desarrollar las relaciones personales positivas, entre otras.

El gran teórico de la Inteligencia Emocional, el psicólogo estadounidense Daniel Goleman, señala que los principales componentes que integran la Inteligencia Emocional son los siguientes:

\section{Autoconocimiento emocional (o autoconciencia emocional)}

Se refiere al conocimiento de nuestros propios sentimientos y emociones y cómo nos influyen. Es importante reconocer la manera en que nuestro estado anímico afecta a nuestro comportamiento, cuáles son nuestras capacidades y cuáles son nuestros puntos débiles. Mucha gente se sorprende de lo poco que se conocen a ellos mismos.

Por ejemplo, este aspecto nos puede ayudar a no tomar decisiones cuando estamos en un estado psicológico poco equilibrado. Tanto si nos encontramos demasiado alegres y excitados, como si estamos tristes y 
melancólicos, las decisiones que tomemos estarán mediadas por la poca racionalidad. Así pues, lo mejor será esperar unas horas, o días, hasta que volvamos a tener un estado mental relajado y sereno, con el que será más sencillo poder valorar la situación y tomar decisiones mucho más racionales.

\section{Autocontrol emocional (o autorregulación)}

El autocontrol emocional nos permite reflexionar y dominar nuestros sentimientos o emociones, para no dejarnos llevar por ellos ciegamente. Consiste en saber detectar las dinámicas emocionales, saber cuáles son efímeras y cuáles son duraderas, así como en ser conscientes de qué aspectos de una emoción podemos aprovechar y de qué manera podemos relacionarnos con el entorno para restarle poder a otra que nos daña más de lo que nos beneficia.

Por poner un ejemplo, no es raro que nos enfademos con nuestra pareja, pero si fuéramos esclavos de la emoción del momento estaríamos continuamente actuando de forma irresponsable o impulsiva, y luego nos arrepentiremos. En cierto sentido, buena parte de la regulación de las emociones consiste en saber gestionar nuestro foco de atención, de manera que no se vuelva contra nosotros y nos sabotee.

Se ha observado que la capacidad de autocontrol está muy ligada a la habilidad a la hora de utilizar el lenguaje: en muchos aspectos, saber gestionar adecuadamente las propias emociones depende de encontrar narrativas que nos permitan priorizar más unos objetivos a largo plazo que otros que tienen que ver con ceder a los impulsos de lo inmediato. Esto encaja con el hecho de que la Inteligencia Emocional comparte tiene mucho en común con la Inteligencia Verbal; tal y como se ha visto al analizar las puntuaciones en pruebas de inteligencia de muchos individuos, ambos constructos psicológicos se solapan mucho.

\section{Automotivación}

Enfocar las emociones hacia objetivos y metas nos permite mantener la motivación y establecer nuestra atención en las metas en vez de en los obstáculos. En este factor es imprescindible cierto grado de optimismo e iniciativa, de modo que tenemos que valorar el ser proactivos y actuar con tesón y de forma positiva ante los imprevistos.

Gracias a la capacidad de motivarnos a nosotros mismos para llegar a las metas que racionalmente sabemos que nos benefician, podemos dejar atrás aquellos obstáculos que solo se fundamentan en la costumbre o el miedo injustificado a lo que puede pasar.

Además, la Inteligencia Emocional incluye nuestra habilidad a la hora de no ceder a las metas a corto plazo que pueden llegar a eclipsar los objetivos a largo plazo, a pesar de que los segundos fueran mucho más importantes que los primeros si nos fueran ofrecidos también a corto plazo (proyectos ambiciosos, planes de ganar mucha experiencia, etc.).

\section{Reconocimiento de emociones en los demás (o empatía)}

Las relaciones interpersonales se fundamentan en la correcta interpretación de las señales que los demás expresan de forma inconsciente, y que a menudo emiten de forma no verbal. La detección de estas emociones ajenas y sus sentimientos que pueden expresar mediante signos no estrictamente lingüísticos (un gesto, una reacción fisiológica, un tic) nos puede ayudar a establecer vínculos más estrechos y du raderos con las personas con que nos relacionamos.

Además, el reconocer las emociones y sentimientos de los demás es el primer paso para comprendere identificamos con las personas que los expresan. Las personas empáticas son las que, en general, tienen mayores habilidades y competencias relacionadas con la IE.

\section{Relaciones interpersonales (o habilidades sociales)}

Una buena relación con los demás es una fuente imprescindible para nuestra felicidad personal e incluso, en muchos casos, para un buen desempeño laboral. $Y$ esto pasa por saber tratar y comunicarse con aquellas personas que nos resultan simpáticas o cercanas, pero también con personas que no nos sugieran muy buenas vibraciones; una de las claves de la Inteligencia Emocional.

Y es que este tipo de inteligencia está muy relacionado con la Inteligencia Verbal, de manera que, en parte, se solapan entre sí. Esto puede ser debido a que parte del modo en el que experimentamos las emociones está mediado por nuestras relaciones sociales, y por nuestra manera de comprender lo que dicen los demás.

Así, gracias a la Inteligencia Emocional vamos más allá de pensar en cómo nos hacen sentirnos los demás, y tenemos en cuenta, además, que cualquier interacción entre seres humanos se lleva a cabo en un contexto determinado: quizás si alguien ha hecho un comentario despectivo sobre nosotros es porque siente envidia, o 
porque simplemente necesita basar su influencia social en este tipo de comportamientos. En definitiva, Ia Inteligencia Emocional nos ayuda a pensar en las causas que han desencadenado que otros se comporten de un modo que nos hace sentirnos de un modo determinado, en vez de empezar pensando en cómo nos sentimos y a partir de ahí decidir cómo reaccionaremos ante lo que otros digan o hagan.

Hay que tomar en cuenta los componentes de la inteligencia emocional ya que estos van a ser el indicio de que se está llevando de manera correcta, primero debemos tener conciencia de nuestras emociones y cómo es que las manejas en el ámbito social; además de ser capaces de generar empatía ante situaciones de adversidad que los otros están viviendo. Ser conscientes que nosotros y los demás tenemos estados de ánimo que pueden sercambiantes y así de esta forma poder tenerun autocontrol emocional generando estrategias para mantener el control en las situaciones de estrés en las que nos podemos ver envueltos y si es que esto nos sobrepasa poder generar actitudes positivas para no perder el control total de la situación.

El poder de con trolar emociones es ideal para el desarrollo de competencias para la vida y lograr un bienestar en el desarrollo conductual.

Ahora bien, si encontramos un fallo en el uso de nuestras emociones, puesto que no hemos logrado aprender a controlarlas, estas nos traerán más problemas pues habrá una mayor desorganización pues el incremento en la frustración por el estrés y esto provocará que la resolución de los problemas sea más y más difícil pues la mente buscará concentrarse en el problema y no en la forma de cómo resolverlos. Tomando en cuenta que el estrés sumado a la frustración y al posible enojo que se va a cumulando genera más tensión y al querer eliminar todas estas emociones podemos actuar de una forma muy negativa y puede que no sólo los afectados seamos nosotros; si no también el resto de las personas que nos rodean y si están involucradas aún más será la descarga hacía ellos y por consiguiente hará el problema más grande de lo que realmente era y como consecuencia habrá una ruptura en las relaciones sociales que se establecieron previamente.

Es importante tomar en cuenta, que además de las consecuencias a nivel social que el mal uso de las emociones nos puede traer, también problemas a nivel orgánico y mental. A nivel orgánico podemos ser acreedores de algunas afecciones como lo son la colitis o gastritis.

"Las emociones tienen una organización compleja en la que interviene una serie de elementos. El estímulo emocional competente (EEC) es el evento que dispara el proceso (de una emoción), y puede originarse en el medio ambiente, de un recuerdo que se evoca al detectar algo similar en el entorno.

Esto lleva al estado de representación, que puede ocumir en cualquiera de las regiones somatosensoriales (visual, auditiva, olfativa, táctil, o la combinación de éstas) y conducir a una activación de sitios ejecutores de respuestas emocionales en el cerebro.

Estas estructuras, disparan la respuesta emocional con la activación de regiones específicas del cuerpo y el reconocimiento de lo que llamamos sentimientos.

Algunas regiones del cerebro identificadas como sitios de activación emocional son las amígdalas, situadas en la profundidad de los lóbulos temporales, además de otras como la corteza motora, que se conoce como cíngulo." (Rafael Salin-Pascual, 2016)

Es importante mencionar que la inteligencia emocional posee una gran carga en el ámbito escolar pues es aquí donde se emplea en muchas ocasiones; además que será una pauta previa para la vida laboral y la forma en cómo nos desenvolvemos.

Para la evaluación de los procesos de aprendizaje, ayudan a identificar las capacidades terminales y los criterios que se pretenden desarrollar. Aunque la evaluación tenga como eje central la comprobación de la adquisición de conocimientos por parte de los alumnos y alumnas, también tendrá en cuenta las actitudes (implicación e interés por la materia y/o actitud ante el trabajo solidario) y los procedimientos (realización de gestiones, de trabajos, ejercicios, tanto en clase como en casa y/o tanto individualmente como en grupo).

Los sentimientos son un sistema de alarma que nos informa sobre cómo nos encontramos, qué nos gusta o qué funciona mal a nuestro alrededor con la finalidad de realizar cambios en nuestras vidas. Una buena percepción implica saber leer nuestros sentimientos y emociones, etiquetarlos y vivenciarlos. Con un buen dominio para reconocercómo nos sentimos, establecemos la base para posteriormente aprender a controlarnos, moderar nuestras reacciones y no dejarnos arrastrar por impulsos o pasiones exaltadas. Ahora bien, ser conscientes de las emociones implica ser hábil en múltiples facetas tintadas afectivamente. Junto a la percepción de nuestros estados afectivos, se suman las emociones evocadas por objetos cargados de sentimientos, reconocer las emociones expresadas, tanto verbal como gestualmente, en el rostro 
y cuerpo de las personas; incluso distinguir el valor o contenido emocional de un evento o situación social.

Se han creado modelos para poder medir el nivel del manejo emocional; se clasifican en 2 grupos: modelos mixtos y de habilidad.

\section{MODELOS MIXTOS}

Aglutinan diversas habilidades, destrezas, objetivos, rasgos de personalidad, competencias y disposiciones afectivas.

a) Modelo de Goleman: Concibe la inteligencia emocional como un conjunto de características clave para resolver con éxito los problemas vitales entre las que destacan: la habilidad de automotivarse y persistir sobre las decepciones; controlar el impulso para demorar la gratificación; regular el humor; evitar trastornos que disminuyan las capacidades cognitivas; mostrar empatía, y generar esperanza.

b) Modelo de Bar-On: La inteligencia socioemocional es un conjunto de competencias y habilidades que determinan cuán efectivamente los individuos se entienden, comprenden a otros, expresan sus emociones y afrontan las demandas de la vida cotidiana.

\section{MODELO DE HABILIDAD}

"Una característica de la inteligencia social que incluye un conjunto de capacidades que explican las diferencias individuales en el modo de percibir y comprender las emociones. Dichas capacidades son las de supervisar los sentimientos y las emociones propias, así como las de los demás, de discriminar entre ellas, y usar la información para guiarel pensamiento y las acciones" Mayery Salovey (1997).

Se derivan cuatro habilidades básicas:

1.- Percepción, valoración y expresión de las emociones. Se relaciona con el uso de las emociones como una parte de procesos cognitivos como la creatividad y resolu ción de problemas, esto se debe a que los estados emocionales dirigen nuestra atención hacia cierta información considerada relevante, determinando tanto la manera en que procesamos la información, como la forma en que enfrentamos problemas.

2.- Facilitación emocional del pensamiento. Esta habilidad se relaciona con el uso de las emociones como una parte de procesos cognitivos como la creatividad y resolución de problemas, esto se debe a que los estados emocionales dirigen nuestra atención hacia cierta información considerada relevante, determinando tan to la manera en que procesamos la información, como la forma en que enfrentamos problemas.

3.- Comprensión de las emociones. La habilidad se refiere al conocimiento del sistema emocional, en otras palabras, a conocer cómo se procesa la emoción a un nivel cognitivo, y cómo afecta el empleo de la información emocional en los procesos de razonamiento; comprende el etiquetado correcto de las emociones, la comprensión del significado emocional, no sólo de emociones sencillas, sino también de emociones complejas, así como la evolución de unos estados emocionales en otros.

4.- Regulación reflexiva de las emociones. Es la que propone el modelo y se relaciona con la capacidad de estar abierto tanto a los estados emocionales positivos como negativos, reflexionar sobre los mismos y determinar si la información que los acompaña es útil sin reprimirla ni exagerar, así como para la regulación emocional de las propias emociones y las de otros.

\section{Conclusión}

En este artículo se plantearon los efectos del control de la inteligencia emocional, cómo influye el mal manejo de las emociones y las repercusiones que se tienen a nivel orgánico y psicológico el no saber emplearlas en el momento oportuno. Además de como con el paso de los años se ha incrementado la importancia de la inteligencia emocional, pues se ha comprobado que la inteligencia no sólo se basa en el desarrollo de habilidades de índole educativo, incluso se ha demostrado que el éxito de una persona no se basa en un ciento por ciento en su IQ, sino que también influye la inteligencia emocional. Las aportaciones de esta investigación buscan mostrar cómo es que la inteligencia emocional, es fundamental para que los alumnos y los profesores puedan entablar ciertas relaciones sin resultar afectados ninguna de las partes al presentarse problemas al establecer empatía. Al igual que va a ayudar al grupo docente a buscar alguna estrategia para que todos los alumnos sean beneficiados de acuerdo con el nivel de manejo de emociones que ellos poseen. Beneficiando que incluso en ellos haya una modificación en los métodos que ellos emplean y así mejorar su desempeño académico.

Por consiguiente, al tener un buen control y desarrollo de las emociones; de esta manera incrementa el desarrollo personal y desarrollo de habilidades educativas.

\section{Referencias}

[1] Operskalski, O. T., Paul, E. J., Colom, R., Barbey, A. K., Grafman, J. (2015). Lesion Mapping the Four-Factor Structure of Emotional 
Intelligence. $\quad$ Front. Hum. Neurosci. doi.org/10.3389/fnhum.2015.00649.

[2] Triglia, Adrián; Regader, Bertrand; y García-Allen, Jonathan (2018). "¿Qué es la inteligencia? Del CI a las inteligencias múltiples". EMSE Publishing.

[3] Goleman, D. (2010). La práctica de la inteligencia emocional. Editorial Kairós.

[4] Rangel, A. E. N. (2014). Inteligencia emocional. Salud vida, 1.

[5] CORREA, R. (2016). las emociones, causa de gastritis, migrañas y colitis. tienen componentes viscerales. gaceta unam (2010-2019), (4814), 8.

[6] Augusto-Landa, J. M., López-Zafra, E. y Pulido-Martos, M. (2011). Inteligencia emocional percibida y estrategias de afrontamiento al estrés en profesores de enseñanza primaria: propuesta de un modelo explicativo con ecuaciones estructurales (SEM). Revista de psicología social, 26(3), 413-425.

[7] Bisquerra, R. (2012). ¿Cómo educar las emociones? La inteligencia emocional en la infancia y la adolescencia. Esplugues de Llobregrat (Barcelona): HospitalSant Joan de Déu.

[8] Borrachero, A.B., Brígido, M., Gómez, R. y Bermejo, M.L. (2012). Relación entre autoconcepto y autoeficacia en los futuros profesores de secundaria. International Journal of Developmental and Educational Psychology: INFAD. Revista de Psicología, 1(2), 219-226.

[9] Borrachero, A. B., Brígido, M., Gómez, R., Bermejo, M. L. y Mellado, V. (2011). Las emociones de los futuros profesores de secundaria sobre el aprendizaje y la enseñanza de las ciencias. International Journal of Developmental and Educational Psychology. INFAD Revista de Psicología, 2(1), 521-530.

[10] Brígido, M. y Borrachero, A.B. (2011). Relación entre autoconcepto, autoeficacia y autorregulación en ciencias de futuros maestros de Primaria. International Journal of Developmental and Educational Psychology: INFAD. Revista de Psicología, 1(2), 107-113.

[11] Ariza-Hernández, M.L. (2017). Influencia de la inteligencia emocional y los afectos en la relación maestro-alumno, en el rendimiento académico de estudiantes de educación superior. Educación y educadores, 20(2), 193-210.

[12] Goleman, D. (1996): Inteligencia emocional. Barcelona, Kairós.

[13] Goleman, D. (1998). Working with Emotional Intelligence. Nueva York: Bantam Books.

[14] Kamphaus, R.W. (2005). Clinical assessment of child and adolescent intelligence. Springer Science \& Business Media.

S. Legg; M. Hutter (2007). "Universal Intelligence: A Definition of Machine Intelligence". Minds and Machines. 17 (4): 391-444.

[15] Vallès, A., y Vallès, C. (2000): Inteligencia emocional: Aplicaciones educativas. Madrid, Editorial EOS.

[16] Bar-On, R. (1997). Emotional Quotient Inventory (EQ-i): A measure of emotionalintelligence. Toronto: Multi Health System.

[17] Brackett, M.A. y Mayer, J.D. (2003). Convergent, discriminant and incremental validity of the competing measures of emotional intelligence. Personality and Social Psychology Bulletin, 29, 11471158 . 